\title{
Statistics of extinction and survival in Lotka-Volterra systems
}

\author{
Guillermo Abramson* \\ International Centre for Theoretical Physics, Strada Costiera 11, P.O. Box 586, 34100 Trieste, Italy \\ Damián H. Zanette ${ }^{\dagger}$ \\ Fritz-Haber-Institut der Max-Planck-Gesellschaft, Faradayweg 4-6, 14195 Berlin, Germany
}

(Received 5 November 1997)

\begin{abstract}
We analyze purely competitive many-species Lotka-Volterra systems with random interaction matrices, focusing the attention on statistical properties of their asymptotic states. Generic features of the evolution are outlined from a semiquantitative analysis of the phase-space structure and extensive numerical simulations are performed to study the statistics of the extinctions. We find that the number of surviving species depends strongly on the statistical properties of the interaction matrix and that the probability of survival is weakly correlated to specific initial conditions. [S1063-651X(98)03204-8]

PACS number(s): 87.10.+e, 82.20.Mj, 02.40.Vh
\end{abstract}

\section{INTRODUCTION}

Systems of interacting biological species evolve through the long, slow, and intricate process of natural selection [1]. Usually, the result of this process is so complex that the dynamics of such webs of coevolving species can be successfully represented, within relatively short time scales, by means of a dynamical system with stochastic elements [2]. A standard mathematical model for the joint evolution of $M$ biological species with spatially homogeneous densities $n_{i}(t)(i=1,2, \ldots, M)$ is the generalized Lotka-Volterra system [3]

$$
\dot{n}_{i}(t)=n_{i}(t)\left[r_{i}-\sum_{j=1}^{M} \kappa_{i j} n_{j}(t)\right] \quad(i=1,2, \ldots, M) .
$$

For large values of $M$, it is reasonable, as a phenomenological approach, to choose the parameters $r_{i}$ and $\kappa_{i j}$ at random from given probability distributions. Within this type of representation, the dynamics of coevolving species can be characterized by statistical properties over different realizations of parameter sets.

There are two biological systems that can potentially involve a large number of coevolving populations. The first one is an ecological system in which each population corresponds to a different biological species, as usually interpreted in the theory of population dynamics [4,5]. The other situation is a system in which each population represents a genotype accessible to a given species [6]. In this situation, the number of populations can be sensibly larger than in the case of interacting species. Although in both cases coevolution is presumably well described by Eq. (1), the probability distributions to be assigned to the random parameters $\kappa_{i j}$, which represent the interaction between populations, are not

\footnotetext{
*Present address: Max-Planck-Institut für Physik Komplexer Systeme, Nöthnitzer Strasse 38, D-01187 Dresden, Germany.

${ }^{\dagger}$ Permanent address: Consejo Nacional de Investigaciones Científicas y Técnicas, Centro Atómico Bariloche, 8400 Bariloche, Argentina.
}

necessarily similar. In fact, in an ecological system of several coevolving species, mutual interactions can be of different types (competition, symbiosis, or parasitism). Within a given species, instead, it is expected that the interaction is mainly competitive, as in logistic models [5].

It is well known that, in a system where many individuals compete for a resource, the dynamics leads to the extinction of some of them and to the survival of others. This is indeed a basic fact of evolution in the Darwinian sense. Though the generalized Lotka-Volterra model (1) has been explored in detail [3,7], it seems that a full characterization, either deterministic or statistical, of the conditions under which a population becomes extinguished or survives in the competition process has not been achieved. In this paper we aim at analyzing this particular problem from a statistical viewpoint.

We consider a large number of coevolving species or genotypes, each of them consisting of a population of identical individuals with density $n_{i}(t)$. These populations are supposed to evolve according to the Lotka-Volterra model (1), subject to purely competitive interactions, i.e., with $\kappa_{i j} \geqslant 0$ for any pair $i, j$. Since we aim at analyzing the statistical properties of the dynamics, these coefficients will be drawn at random from a given distribution and will remain quenched from the initial time.

For simplicity, we take $r_{i}=1 \forall i$ [2], indicating that in the absence of competition the dynamics of all the populations are identical. We are thus implicitly identifying these populations with the genotypes accessible to a given species. Within this condition, that it is not essential to our interest and could in fact be easily relaxed, Eq. (1) reduces to

$$
\dot{n}_{i}(t)=n_{i}(t)\left[1-\sum_{j=1}^{M} \kappa_{i j} n_{j}(t)\right] \quad(i=1,2, \ldots, M) .
$$

All the coefficients $\kappa_{i j}$ will be chosen at random from the same distribution $p(\kappa)$, such that $p(\kappa)=0$ for $\kappa<0$.

In the next section we outline the behavior of the dynamical system (2) in phase space, showing that its evolution proceeds along a series of "pseudoextinctions," in which some of the densities $n_{i}(t)$ can attain very low levels during long periods but, eventually, they recover significative val- 
ues. A threshold for these pseudoextinctions, which become consequently true extinctions, is suggested by the biological context of the problem. This threshold is introduced in our numerical study of Eq. (2) in Sec. III, where we focus the attention on the statistics of extinct and surviving genotypes and try to characterize their long-time behavior in terms of their inital conditions. Our results are discussed in Sec. IV.

\section{PHASE-SPACE STRUCTURE}

The evolution of the dynamical system (2) can be described in terms of a semiquantitative analysis of the corresponding phase-space topology, which is determined by the fixed points of Eq. (2) and the associated invariant manifolds. The equation for the fix-point coordinates $n_{i}^{*}$ reads

$$
n_{i}^{*}\left(1-\sum_{j} \kappa_{i j} n_{j}^{*}\right)=0 \quad(i=1,2, \ldots, M)
$$

and has, generically, $2^{M}$ solutions. In fact, each solution to this equation can be characterized by the number $M^{\prime}$ of nonzero coordinates $\left(M^{\prime}=0, \ldots, M\right)$; let us call such a solution an $M^{\prime}$ equilibrium. For a given choice of the coefficients $\kappa_{i j}$ the number of different $M^{\prime}$ equilibria is $C\left(M, M^{\prime}\right)$ $=M ! / M^{\prime} !\left(M-M^{\prime}\right) !$. Therefore, disregarding pathological choices of $\kappa_{i j}$, the total number of fixed points is $\Sigma_{M^{\prime}} C\left(M, M^{\prime}\right)=2^{M}$.

Since $n_{i}^{*}$ stands for a density, meaningful equilibria among the $2^{M}$ fixed points are those with non-negative coordinates. In the Appendix it is proved that, for random $\kappa_{i j}$, the probability that all the nonzero coordinates of an $M^{\prime}$ equilibrium $\left(M^{\prime} \neq 0\right)$ are positive is

$$
P\left(M^{\prime}\right)=2^{1-M^{\prime}} .
$$

We stress that it is essential to this result that $\kappa_{i j}>0 \forall i, j$, i.e., that the system is purely competitive. For large $M$, the number of equilibrium points with non-negative coordinates will therefore be approximately given by

$$
\sum_{M^{\prime} \neq 0} 2^{1-M^{\prime}} C\left(M, M^{\prime}\right) \approx 2\left(\frac{3}{2}\right)^{M} .
$$

It is interesting to note that, if $\kappa_{i j}>\kappa_{\min } \forall i, j$, all the nonnegative equilibria will be confined to a certain volume $V$ in phase space since $\Sigma_{i} n_{i}^{*}<1 / \kappa_{\min }$. This volume shrinks rapidly for growing $M$, as $V=\kappa_{\min }^{-M} / M$ !, and the density of equilibrium points, most of which are situated on the surface of $V$, where some of the coordinates vanish, grows correspondingly.

The stability properties of the fixed points of system (2) can be fully analyzed in some very special cases only. For instance, as could be expected for this logisticlike dynamical system, the 0 equilibrium $\left(n_{i}^{*}=0 \forall i\right)$ can be proved to be always unstable. Moreover, for a random choice of positive $\kappa_{i j}, 1$ equilibria are stable with probability equal to $M^{-1}$. Finally, the $M$ equilibrium $\left(n_{i}^{*} \neq 0 \forall i\right)$ is stable if $\kappa_{i j}$ is a symmetric matrix.

Though we cannot give a detailed characterization of the stability of all of the $M^{\prime}$ equilibria, it can be argued that, for a random system and for large $M^{\prime}$ and $M$, the eigenvalues of the linearized problem should follow a semicircular distribution [8]. A typical equilibrium point is thus linearly unstable and it has approximately the same number of positive and negative eigenvalues. Correspondingly, the number of unstable and stable invariant manifolds for each equilibrium is more or less the same. Since the mathematical structure of system (2) prevents both the divergence of orbits and changes of sign in the densities $n_{i}(t)$, the invariant manifolds of positive equilibria are necessarily bounded and mutually connected, defining homoclinic and heteroclinic orbits. Most of these orbits lie on the surface of the volume that contains the positive equilibria, where some of the densities are exactly equal to zero.

In summary, the portion of the $M$-dimensional phase space of system (2) meaningful to our problem is populated by a large set of fixed points, of the order of $(3 / 2)^{M}$ in number, most of them having positive and negative eigenvalues, i.e., being unstable. They are confined to a volume of order $1 / M$ ! and typically are found on the surface of such volume. These equilibria are highly interconnected through invariant manifolds that lie also on that surface and connect stable and unstable eigenvectors. The number of those manifolds should be of order $M(3 / 2)^{M}$.

With these elements in hand, the evolution along a typical phase-space trajectory of the dynamical system (2) can be outlined as follows. From a generic initial condition, the orbit should soon approach one of the stable manifolds and the system will be driven towards the corresponding equilibrium. It will spend some time in the vicinity of this equilibrium, but if this fixed point is not stable (i.e., if it has at least one unstable manifold, which, as we have argued, is the typical case) the orbit will finally leave that neighborhood, just to be drawn along one of the unstable manifolds of this first equilibrium point towards another equilibrium, which is expected to have in turn some stable and some unstable manifolds. The whole process will repeat itself and the system will wander in phase space, typically visiting the neighborhoods of a large number of unstable fixed points, until it eventually finds a stable equilibrium. This is reminiscent of the complex behavior of Boolean evolution models on random landscapes [9], which, in contrast to Lotka-Volterra models, are discrete (in space and time) and stochastic.

We stress that, in wandering from one equilibrium to another, the orbit is expected to approach more and more the successive invariant manifolds that drive the dynamics of the system [10]. This implies, in particular, that the system will spend longer and longer periods in the immediate vicinity of those equilibria. Since typically the equilibria have some null coordinates, the corresponding densities will approach a vanishing state but, as the system escapes from each unstable equilibrium point, they can eventually recover appreciable values. As the evolution proceeds, a given density can therefore practically vanish during a rather long time, but can then increase and become again significant in the whole dynamics. We shall return to these pseudoextinctions in the following section to discuss their relevance in the numerical study of the system and its biological interpretation.

Finally, it is worthwhile to remark that the existence of a stable equilibrium point, able to definitively attract an orbit, is in principle not guaranteed. Moreover, even if one or several stable points do exist, it is not ensured that their basins 


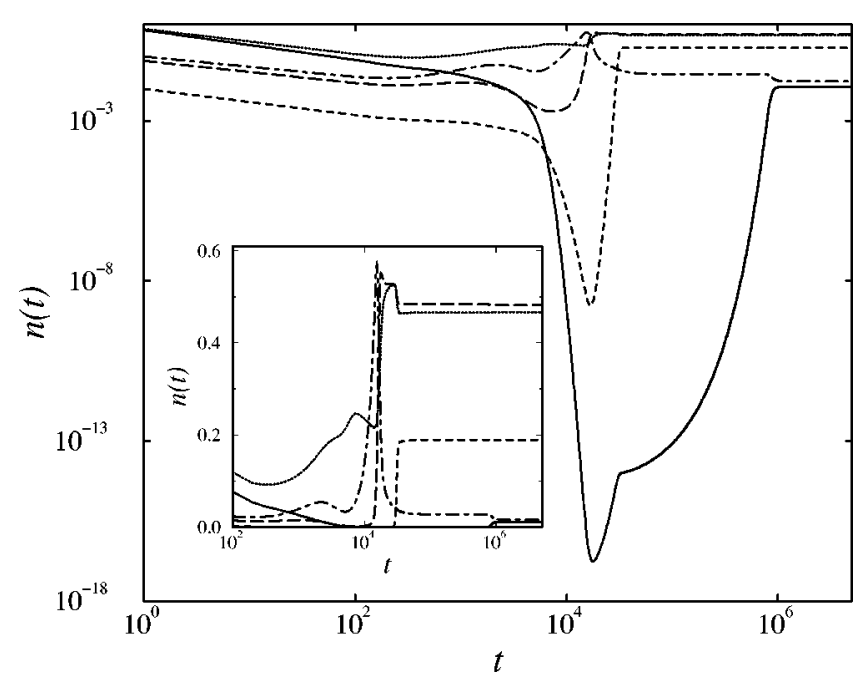

FIG. 1. Time evolution of the density of some selected populations, from a system consisting of 20 populations. Both axes are logarithmic to emphasize how some of the populations, after becoming almost extinct, grow again to significative values. Inset: the same curves in a log-linear plot.

of attraction cover the whole space of initial conditions. The system could thus perform a chaotic orbit or become trapped in a limit cycle $[10,11]$.

\section{NUMERICAL ANALYSIS}

We have performed an extensive numerical investigation of system (2). Each realization consists of the numerical integration of the equations, after a random choice of the interaction matrix and the initial conditions. In all the results presented here, the interaction coefficients $\kappa_{i j}$ have been randomly chosen from a uniform distribution in the interval $\left[\kappa_{0}-\Delta \kappa, \kappa_{0}+\Delta \kappa\right]$, with $\Delta \kappa \leqslant \kappa_{0}$, but we have tested that other probability distributions, always defined for $\kappa>0$, produce essentially the same results. Similarly, the initial densities have been uniformly distributed at random in the interval $\left[0, n_{\max }\right]$. A proper rescaling of densities and time makes it possible to fix, without generality loss, $\kappa_{0}=1$ and $n_{\max }=1$. The only parameter to vary in these distributions is therefore $\Delta \kappa$. In the following we describe the dynamical behavior of Eq. (2) as drawn from our numerical calculations.

\section{A. Pseudoextinctions and density threshold}

In Fig. 1 we show the evolution of several typical densities in a system of $M=20$ genotypes for $\Delta \kappa=0.5$. Note that, to ease the appreciation of certain details, both the time and the density axes are logarithmic. In the inset the same curves are shown in a semilogarithmic plot, with a logarithmic time axis. The phenomenon of pseudoextinctions is clearly seen in some of the curves. We have checked that, in some realizations, one or more densities can temporarily attain values as small as $n \sim 10^{-16}$ and then grow to levels of the order of their initial values. The verification that pseudoextinctions do occur, as predicted, in Sec. II, from our analysis of the phasespace structure, points the attention to another factor, which is not present in the model as described by Eq. (2), but has to be necessarily taken into account in a system where the variables are actually discrete. In fact, the population density of a

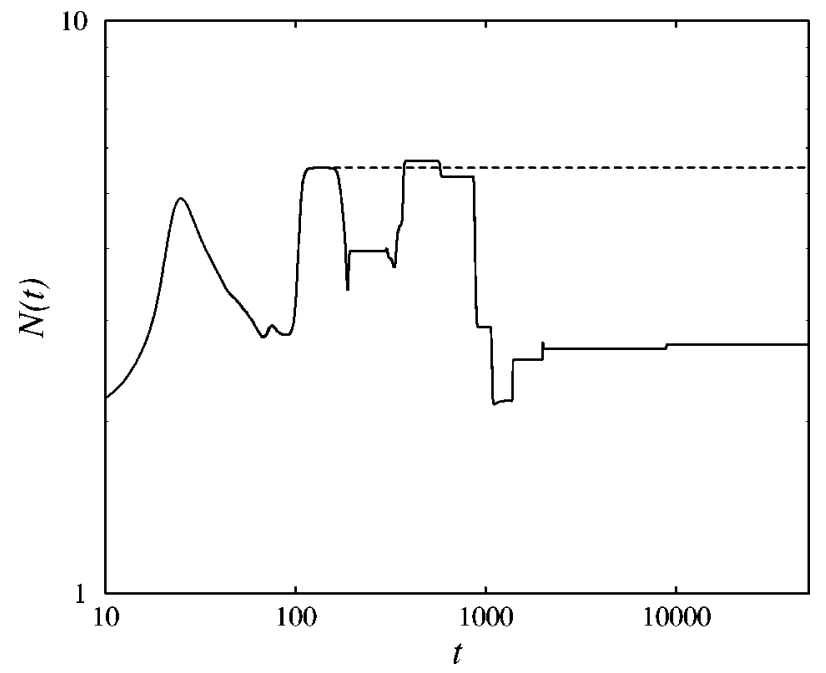

FIG. 2. Time evolution of the total density. Full line, the system without a density threshold; dashed line, the same system with a density threshold. Note the logarithmic scale in the time axis.

species or a genotype confined to certain spatial domain $\Omega$ of volume $V_{\Omega}$ cannot be smaller than $V_{\Omega}^{-1}$, unless it vanishes. In a description in terms of densities, it is therefore necessary to fix a threshold [12], below which the only value accessible to the density is effectively zero. In addition to this biological argument for introducing a density threshold, we must stress that in our numerical calculations this element is also necessary to avoid spurious effects of finite computer precision on the results. A threshold $n_{0}$ thus has been introduced as an additional parameter in the numerical calculations, in such a way that if a density attains a value lower than $n_{0}$ it is automatically set to zero.

From the analytical viewpoint, it can be argued that the introduction of a threshold changes the stability of almost all the $M^{\prime}$ equilibria. Roughly speaking, whereas without a threshold an orbit could approach an equilibrium following a stable manifold just to leave it along an unstable one, with a threshold the system can instead be "captured"' by the equilibrium point if the orbit crosses the threshold. What was an unstable equilibrium becomes, in effective terms, a stable one.

In order to illustrate the different behavior of systems with and without density threshold, we have chosen to analyze the evolution of the total density

$$
N(t)=\sum_{i=1}^{M} n_{i}(t)
$$

as a global characterization of the phase-space dynamics. Figure 2 displays the evolution of $N(t)$ for two systems of 100 species. In both cases the interaction is defined by $\Delta \kappa=1$. One of the curves corresponds to the system without a threshold (in addition to that imposed by the smallest representable number in the computer). The other one corresponds to the same system, with the same initial conditions and interaction matrix, with a threshold $n_{0}=10^{-6}$. Note that the time scale is again logarithmic. It can be seen that, in this realization, the orbit of the first system follows the qualitative behavior described in Sec. II. It passes near some equilibria, where $N(t)$ remains practically constant, spending ex- 


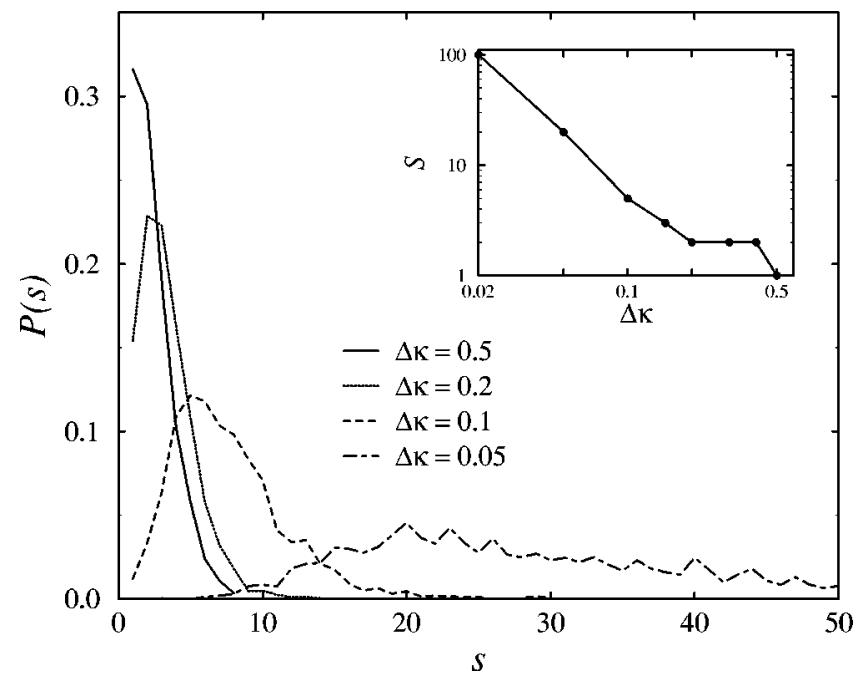

FIG. 3. Distribution of the number of survivors $P(s)$. Each curve corresponds to 2000 realizations of systems with $\Delta \kappa$ as shown in the legend. Inset: the position of the maximum of the distribution $S$ as a function of the width $\Delta \kappa$ of the distribution $p(\kappa)$.

ponentially longer and longer times in their neighborhood. When a threshold is present, the system is always attracted to one of the new "stable" equilibria. In the case of Fig. 2, the system is captured at $t \approx 100$ by an equilibrium point that, although being unstable in the first case, acts now as a stable fixed point for this orbit.

\section{B. Statistics of survivals}

According to our simulations, the main feature in the long-time dynamics of system (2), with or without a threshold, is that it evolves to a situation in which most of the densities vanish, at finite times if $n_{0} \neq 0$ or asymptotically if $n_{0}=0$. This behavior can be identified with the extinction of the corresponding genotypes. In any case, for a large system, a variable number of surviving populations is found at long times. In Fig. 3 we show the distribution of the number of surviving populations for different values of $\Delta k$. Each curve was constructed from the results of 2000 realizations in a system with $M=100$ and $n_{0}=10^{-6}$. The final time in each realization was chosen so that a stationary state had been reached, which was checked to be a solution to Eq. (3).

The distribution of the number of surviving populations is generally a bell-shaped curve, its width and maximum depending on the probability distribution $p(\kappa)$. It can be seen in Fig. 3 that for $\Delta \kappa$ small enough the curve is relatively broad and that wider interactions reduce the overall stability of the system, leading to a shift of the curve towards a situation where fewer species survive. The correlation between the maximum of the distribution of survivors and the width of $p(\kappa)$ is shown in the inset, in a log-log plot. Observe that for the smallest value $\Delta \kappa=0.02$ the maximum of the distribution coincides with the total number of species in the system.

It is worthwhile to note that the probability of having a non-negative $M^{\prime}$ equilibrium $P\left(M^{\prime}\right)=2^{1-M^{\prime}} C\left(M, M^{\prime}\right)$ (cf. Sec. II) is also a bell-shaped curve as a function of $M^{\prime}$. This fact, however, should be considered only as indicative of the



FIG. 4. Probability of survival of a single species $P_{s}$ as a function of its initial density $n_{0}$. The two curves correspond to 2000 realizations of a symmetric and an asymmetric system.

profile of the curves in Fig. 3. In fact, the probability that an initial condition approaches any $M^{\prime}$ equilibrium depends not only on their number for a given $M^{\prime}$ but also on the size of their basins of attraction, about which our phase-space analysis provides no information.

\section{Characterization of survivors}

A very natural question arises now, yet not easy to answer: Which populations survive? Are they characterized by some particular initial condition, by some peculiarity in the interaction with the remaining populations? In the study of a real ecological system, it would be desirable to give an answer to these questions in terms of quantities accessible from observations. It is thus reasonable to consider, since they are probably the easiest to measure, the initial densities and their initial time derivatives. In addition to being accessible, these quantities characterize the initial interactive scenario: According to Eq. (2), the density measures the effects of each population on itself, while its first time derivative accounts for the influence of the remaining species.

We have found that an answer based on deterministic arguments cannot be given to such questions. According to the statistics collected from the simulations, we conclude that only a weak correlation exists between survival and the initial conditions. This correlation can be evidenced by calculating the distribution of final densities as a function of the initial one, thus providing a probabilistic answer to those questions. In Figs. 4 and 5 we show (full lines) the distribution of survivors as functions of initial values, for general asymmetric systems $\left(\kappa_{i j} \neq \kappa_{j i}\right)$. Figure 4 shows that the probability of survival is almost uniform in the whole range of initial densities, with a slighty higher probability of survival for the largest ones. In Fig. 5 an associated distribution is shown: the number of survivors as a function of the initial derivative of the density. Here an enhancement of the probability of surviving is seen around a relatively large (and negative) value of the initial time derivative.

We have also found that this correlation between the final state and the initial condition is stronger in symmetric sys- 


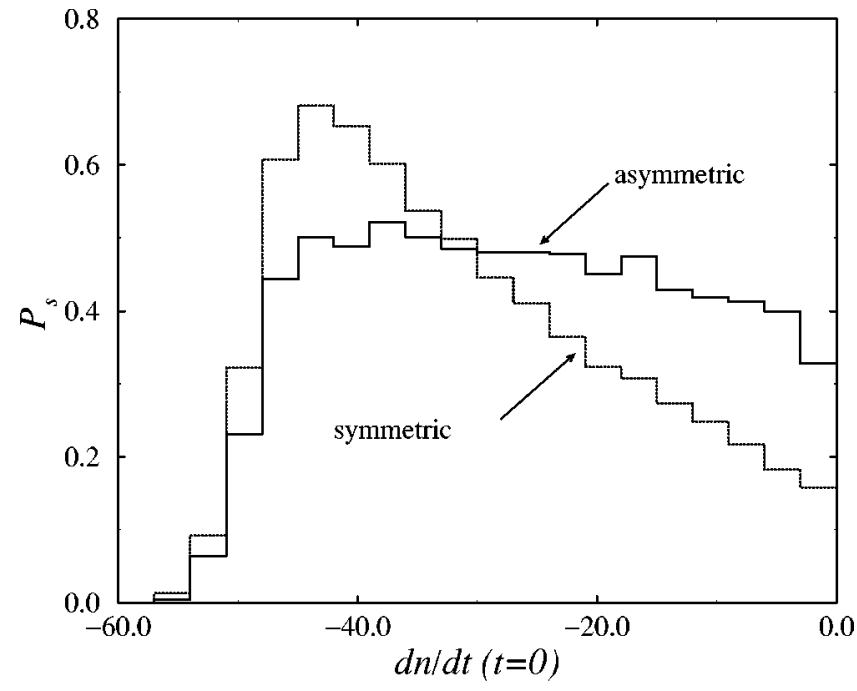

FIG. 5. Probability of survival $P_{s}$ as a function of the initial derivative of the density $d n /\left.d t\right|_{t=0}$. The two curves correspond to 2000 realizations of a symmetric and an asymmetric system.

tems $\left(\kappa_{i j}=\kappa_{j i}\right)$. In Figs. 4 and 5 the same functions are shown as dashed lines for symmetric systems. In this case there is an enhancement in the probability of survival for those species that start with a higher initial population. Although considering a purely symmetric interaction matrix is irrelevant from a biological point of view, these results suggest that the statistical correlation between initial and final states and, in particular, between initial conditions and survival probability can depend in a rather strong manner on additional constraints in the interaction matrix.

\section{CONCLUSIONS}

We have analyzed a dynamical system that represents the evolution of many species coupled by Lotka-Volterra interactions. The study has been restricted to systems where the interaction is purely competitive. This dynamical system describes, in principle, two different biological systems. The first is an idealized ecological system of interacting species. To represent more realistic ecological systems, the connectivity of the model should be built correspondingly, typically, with several levels of preys and predators.

On the other hand, the model can also describe the system of genotypes present in, or accessible to, a single species or population. Within a single species, the number of competing genotypes can be much larger than the number of competing species in an ecological niche. Of course, not all of them strive. The surviving genotypes are finally expressed in the living population. This is precisely the problem we have addressed in this paper.

We have found that the evolution of the system follows complicated orbits in phase space. These orbits drive the system from the neighborhood of one of the many equilibria to another, regardless of their stability. Systems with a finite population threshold, which may represent more accurately real biological systems, eventually fall into a stable equilibrium situation. As time elapses, a variable number of populations become extinct through the interaction with the others. In general, more than one species survive, in contrast with the "principle of competitive exclusion" [5] (which is known to be of limited validity). The number of surviving species, those that finally reach equilibrium, is characterized by a bell-shaped distribution whose width and maximum depend on the distribution of interactions.

In addition to competition, a population of genotypes is also subject to changes that arise from random mutations and recombination during the reproduction of the organisms [13]. The description of such a system would require a modification of the model, whose behavior cannot be predicted $a$ priori. Mutations can be easily taken into account by allowing a different interaction, namely, random transitions between the genotypes [14]. The analysis of this system is the subject of work in progress.

\section{ACKNOWLEDGMENTS}

The authors are grateful to D.E. Strier and S.C. Manrubia for enlightening discussions. D.H.Z. thanks the Alexander von Humboldt Stiftung for financial support and the Fritz Haber Institut for hospitality during his stay in Berlin. Financial support from Fundación Antorchas, Argentina, is also acknowledged.

\section{APPENDIX}

Theorem. Let $\left\{\kappa_{i j}\right\}$ be an $M \times M$ random matrix, whose coefficients are drawn from the same probability distribution $p(\kappa)$, with $p(\kappa)=0$ for $\kappa \leqslant 0$. Then the probability that the solution to the set of linear equations

$$
\sum_{j=1}^{M} \kappa_{i j} n_{j}=1, \quad(i=1,2, \ldots, M)
$$

has positive components $n_{i}>0 \quad \forall \quad i=1,2, \ldots, M$ is $P=2^{1-M}$.

Proof. For $M=1, n_{1}=1 / \kappa_{11}$, which is always positive $(P=1)$. For $M=2$, the system can be explicity solved and in particular we get

$$
\frac{n_{1}}{n_{2}}=\frac{\kappa_{22}-\kappa_{12}}{\kappa_{11}-\kappa_{21}} .
$$

The symmetry of this expression with respect to the coefficients $\kappa_{i j}$ makes it clear that $n_{1} / n_{2}>0$ with probability $1 / 2$, irrespectively of the form of $p(\kappa)$. Now, since $\kappa_{i j}>0 \forall i, j$, if $n_{1}$ and $n_{2}$ have the same sign and satisfy Eq. (A1) with $M=2$, they must be positive. Therefore, $P=1 / 2$.

For $M>2$, we take any pair of equations from Eq. (A1), say, the $k$ th and the $l$ th, and rewrite them as

$$
\begin{gathered}
\kappa_{k k} n_{k}+\kappa_{k l} n_{l}=1-\sum_{j \neq k, l} \kappa_{k j} n_{j}, \\
\kappa_{l k} n_{k}+\kappa_{l l} n_{l}=1-\sum_{j \neq k, l} \kappa_{l j} n_{j} .
\end{gathered}
$$

This can also be put in the form

$$
\begin{gathered}
\kappa_{k k}^{\prime} n_{k}+\kappa_{k l}^{\prime} n_{l}=1, \\
\kappa_{l k}^{\prime} n_{k}+\kappa_{l l}^{\prime} n_{l}=1,
\end{gathered}
$$


with $\kappa_{k k}^{\prime}=\kappa_{k k} /\left(1-\sum_{j \neq k, l} \kappa_{k j} n_{j}\right)$ and analogous expressions for $\kappa_{k l}^{\prime}, \kappa_{l k}^{\prime}$, and $\kappa_{l l}^{\prime}$.

Note that for fixed, arbitrary values of $n_{j}(j \neq k, l)$, the functional form of the primed coefficents in terms of the original ones is the same. The probability distributions for $\kappa_{k k}^{\prime}, \kappa_{k l}^{\prime}, \kappa_{l k}^{\prime}$, and $\kappa_{l l}^{\prime}$ are therefore identical. Hence, as a consequence of the previous result for $M=2$, the probability that $n_{k} / n_{l}$ is positive equals $1 / 2$. This holds irrespectively of the distribution for the primed coefficients, i.e., irrespectively of the values of $n_{j}(j \neq k, l)$. The relative sign of any two

[1] P. Skelton, Evolution: A Biological and Paleontological Approach (Addison-Wesley, Wokingham, 1994).

[2] R.M. May, Nature (London) 238, 413 (1972).

[3] Yu.M. Svirezhev and D.O. Logofet, Stability of Biological Communities (Mir, Moscow, 1983).

[4] A.S. Mikhailov, Foundations of Synergetics I (Springer, Berlin, 1990), Chap. 7.

[5] J.D. Murray, Mathematical Biology (Springer-Verlag, Berlin, 1993).

[6] P. Schuster, Physica D 22, 100 (1986).

[7] R.M. May, Stability and Complexity in Model Ecosystems (Princeton University Press, Princeton, 1974). components $n_{k}$ and $n_{l}$ of the solution to Eq. (A1) is then statistically independent of the values of the other components.

To ensure the positivity of all the components it is sufficient to consider $M-1$ ratios $n_{k} / n_{l}$, for instance, $n_{1} / n_{l}$ with $l=2,3, \ldots, M$. According to the above results, the probability that all these ratios are positive is $(1 / 2)^{M-1}$. Now, since $\kappa_{i j}>0 \forall i, j$, if all the $n_{i}$ have the same sign and satisfy Eq. (A1), they must be positive. Therefore, $P=(1 / 2)^{M-1}$ $=2^{1-M}$.
[8] C.E. Porter, Statistical Theories of Spectra: Fluctuations (Academic, New York, 1965).

[9] S.A. Kauffman, Physica D 42, 135 (1990); J. Theor. Biol. 149, 467 (1991).

[10] A.S. Mikhailov and I.Yu. Poteryaiko, Physica D 53, 13 (1991).

[11] P.E. Phillipson and P. Schuster, J. Chem. Phys. 79, 3807 (1983).

[12] L.S. Tsimring, H. Levine, and D.A. Kessler, Phys. Rev. Lett. 76, 4440 (1996).

[13] M. Eigen, Naturwissenschaften 58, 465 (1971).

[14] T. Boseniuk, W. Ebeling, and A. Engel, Phys. Lett. A 125, 307 (1987). 